\title{
A systematic innovation case study: new concepts of domestic appliance drying cycle
}

\author{
S. Graziosi ${ }^{1}$, D. Polverini ${ }^{2}$, P. Faraldi ${ }^{3}$, and F. Mandorli ${ }^{4}$ \\ ${ }^{1}$ Università Politecnica delle Marche, Mechanical Engineering Department, Italy, \\ s.graziosi@univpm.it \\ ${ }^{2}$ Università Politecnica delle Marche, Mechanical Engineering Department - \\ Indesit Company, Innovation \& Technology division, Italy, \\ davide.polverini@indesit.com \\ ${ }^{3}$ Indesit Company, Innovation \& Technology division, Italy, \\ paolo.faraldi@indesit.com \\ ${ }^{4}$ Università Politecnica delle Marche, Mechanical Engineering Department, Italy, \\ f.mandorli@univpm.it
}

\begin{abstract}
While incremental innovation is for most companies a well assessed process, radical product innovation is often handled with difficulty, mainly due to myriad obstacles in the idea-to cash process which limits company's ability to innovate. As a typical approach, engineers firstly try to find innovative solutions only inside their technological product space, basically thinking accordingly to their commonly assessed know-how. In this paper an industrial case is analyzed, showing how TRIZ methodology offers to technicians a systematic way to solve problematic contradictions and find effective ideas.
\end{abstract}

Keywords: Radical Innovation, Concept Design, TRIZ, Idea Generation.

\section{Introduction}

Innovation is a complex process, of dramatic importance for organisational success. Due to international competition intensification and product development process shortening, the pressure to innovate has continuously been heightening since the last decades.

The enterprise need for innovation is clearly expressed by Porter and Stern [1] considering innovation as "the transformation of knowledge into new products, processes, and services - involves more than just science and technology. It involves discerning and meeting the needs of the customers". The terms "innovation" has been deeply analyzed in scientific literature, mainly focusing on the difference between incremental innovation and radical one. Kim and 
Mauborgne [2] consider incremental innovation as a sort of "imitation" while companies should focus on proactive strategy aiming at creating new customers and markets as well as sustaining the existing ones. On the contrary, author such as Harrington [3] argue that continuous improvements are the major driving forces without which the gains for radical improvements are not sustainable. Our point here is that both incremental and radical innovations are really fundamental for company growth. More than in incremental innovations, in radical ones engineers and technicians should extend their creativity on finding the best technical solutions to drastically and positively change product day-life image. However, in some cases all the attempts at finding these solutions are really stressful and not always successful because of "psychological inertia" which persuades engineers on reasoning only inside their own paradigm or "enterprise area". As S.D. Savransky said [4], psychological inertia "leads us away from the solution of the problem, impedes problem recognition and clarification, creates barriers during the search for step to solution, complicates decision making, and makes other stage of the problem solving difficult". New methodological approaches are needed [5], allowing to effectively start designing process from a general model of the problem, efficiently converging towards the best technical solutions.

In this regard, the present paper aims at showing how TRIZ theory implementation allows the access to the best methodologies and instruments to stimulate and discipline thinking activity leading to really effective problem solutions. An industrial case has been analyzed, where a standard, assessed technology needs to be renewed to comply with emerging boundary conditions in terms of customer needs and regulations: this study case is the drying phase of domestic appliances such as dishwashers, dryers and wash-and-dryers. The experimental tests have been carried on in collaboration with Indesit Company, an important European company, leader in the domestic appliances market. Over the last years, a strong focus on innovation has been performed by this company: a dedicated technical area, the Innovation\&Technology Direction, has been built up, whose specific aim is to constantly bring innovation into the industrial products: new technologies, new materials, breakthrough product concepts are continuously being investigated, in order to assess their technical feasibility on the domestic appliance environment.

In this article, we firstly overview which are the main driving innovation forces in modern enterprises, focusing on household appliances, and how firms try to enrich these outcomes. Section 3 describes the drying cycle and the company need for innovation. In Section 4 we discuss our approach to the problem using TRIZ Contradictions Matrix. 


\section{Driving innovation forces in modern enterprises}

Product innovation in industrial process and design of consumer goods is nowadays increasingly achieving more and more importance: continuous strengths are held in order to maintain product competitiveness at top level, to efficiently face competitors' actions and increase the market share. Systematic methods to be used in the day-by-day activity are still not present in standard product life cycle management, but a continuously increasing number of innovation projects, aimed to generate new product concepts, are now common also in this industrial sector. "Market-driven projects" and "technology-driven projects" are two typical cases of new concepts projects. In both cases, two basic instruments are used: brainstorming sessions among researchers, designers, marketing people, and consumer tests. Two are the key points for innovation: a clear identification of customer and market needs and the transformation of these requirements into innovative technical solutions. Preliminary actions shall be mainly focused in correctly understanding the real likes and dislikes of potential customers, and also in assessing the added value they would be ready to charge in the new product. The problem here is that consumer test results are often unclear, and the more innovative is the concept, the harder is to obtain an immediately enthusiastic answer among all consumers. Leaving out these marketing aspects, in this paper we focus on engineering ones, or better we implement a methodology useful to translate marketing aspect into technical solutions.

As above mentioned the first technical idea came out during brainstorming sessions, being these ones a method of creativity activation, in order to overcome psychological inertia [6]. Developed by Alex Osborn in the 1940's, brainstorming consist of an initial analysis of the situation by means of a list of central questions, and a work phase during which technicians generate an idea and than critique it, as recommended by classical philosophical theories. This method, as A. Orloff said [7], has some advantages because is easy to learn and use, and relatively little time is needed to prepare a session; also idea generation process usually ends with positive results, even if it is not well structured. As assessed by technical and scientific literature, the higher the problem difficulty, the less effective is the solution by using this method, which in the end seems not to be capable of stimulating inspiration. The results are often long and helpless searching: considerable material and intellectual effort, weak and useless ideas and useless deviations from potentially useful goals. In this regard there is a need for the enterprise to introduce new methods of idea generation which can "help control thinking for solutions to construction-technical problems with extreme physical and technical contradictions. Concrete constructive navigators are needed for concrete problematic situations." [6].

To overcome all these drawbacks, the company is willing to experiment another strategy of work in household appliances innovation projects: TRIZ theory has been chosen. The firm interest for TRIZ is due to its ability on carrying on idea generation in a well-framed way and stimulating inventiveness: "the primary 
goal of TRIZ is to help thinking become talented and intuition become controllable, structured, and functioned well" [7]. TRIZ theory can help companies on eliminating typical mistakes deriving by incorrectly structured inventing sessions, such as extreme concreteness of problem description or lack of a general model as a starting point to analyze a specific industrial problem. Engineers would be hopefully supposed to change their usual approach to technical problem solving, being able to easily "sketch" a general model as an abstraction of the original technical issue. It equips the "user" with a clear understanding of the initial situation and of all the problem solving and idea generation stages, giving a wider vision of all the aspect involved. Moreover company interest is also focused on achieving a better and effective use of patent literature analysis because nowadays, it is usually performed at further project steps, to assess if a specific technical solution can be patented or if other inventors have already developed similar ideas.

\section{The household appliances innovation forces: the drying cycle}

Drying process today represents a typical phase of domestic appliances cycles standard sequence, mainly dishwashers, wash-and-dryers and dryers; the aim is to dewater humid items like clothes or crockery, which underwent previous washing phases, to targeted values of residual in order to restore initial conditions. Different technical solutions can be implemented to reach this target, having always basically in mind item care on one side, and process duration on the other. Potential improvements on drying phase could be very appealing for final consumers; the following list shows the most common features, parameters, criticisms:

1. Energy consumption, up to $40-50 \%$ of total cycle for wash-and-dryers;

2. Up to $40 \%$ of total cycle duration of dishwashers;

3. Handling operations of garments load to be repeated twice for a consumer that washes clothes in a washing machine and dries the same ones in a dryer;

4. Inhomogeneous distribution of dried load final humidity;

5. Undesired effects for consumers, e.g. the "fog" coming out of the dishwasher tub when door is open at the end of the cycle;

The focus of the present analysis has been the energy consumption reduction and the decrease of the drying cycle duration as primary issues, having taken as a basic reference the results of preliminary consumer tests, specifically held to deeply understand final user's point of view. Another interesting and surprising advice, emerged from the study, is that "ready for use" clothes are not so appealing for the majority of consumers: always having in mind garments care, a specific cycle aimed to eliminate further ironing operations would be perceived as a "niche" function to be used only on low quality garments. 
Another important issue, which then reflects in further constraints, is the design and engineering of the appliance. More in details, it means that any innovation to be implemented on the product to satisfy the target (time decrease, energy consumption decrease and "ready to use" garments) shall not: dramatically increase product industrial cost, heavily affect currently assessed industrial process and decrease product reliability. It is anyway evident that the more innovating and performing is the concept, the heavier modifications will be accepted on the design and on the production process. For a completely breakthrough concept, a brand-new design and process could be hypothesized, but a trade-off between new performance and product changes is usually reached. No trade off is accepted on product reliability: first of all, on large-scale industrial production "low quality costs" are already an important issue to control both, from the financial and brand image point of view, so that no unreliable product, even if genial in its innovation, would be accepted. Moreover, people's health and safety while using the appliance is a "must" to be respected in any case.

In the paper test case, traditional abovementioned drivers such as cost, quality or process optimization are not suitable driving forces, because something more radical is needed: a significantly increase on current product performance, new performance and new product design and styling, in order to better fit the desired target of creating totally new products.

\section{Test Case}

\subsection{The approach}

By considering a generic tool, designed to clean items, whether they are garments or crockery, it can be easily acknowledged that one of the most common technical approach is based on using a solution of water and detergent, with which items are put in direct contact. As a brief description of the process, stain removal is mainly due to detergent action, whilst water results in being the carrier of the stain particles, from the item to the waste system (a deeper analysis of chemical and physical phenomena would not be consistent with the target of the present paper). In any case, the target of the dedicated tool can be set as "restoring the initial conditions" of a clean and dry item. Accordingly to this hypothesis, two basic operation modes will be identified: a detergent water activation mode (washing or rinsing) and a water extraction mode. From the TRIZ point of view, for domestic use washing machine (the technique), the product primary function is related to the first operation, while the water extraction can be considered as auxiliary because it ensures the accomplishment of the primary one. It is not worthless to underline that in other household appliances like dryers, the washing operation does not exist and the primary function is represented by the water extraction 
itself. Dryers are mainly used to perform clothes drying phase in countries whose weather conditions can not ensure an appropriate environment. It is not a case that the implemented drying phase technical solution reflects what happens in nature, using hot flow hair which circulates inside the tank. Thanks to the thermal convection and heat conduction the water changes its physical state and can be removed from clothes. In a washing machine the elimination of water is achieved only by mechanical centrifugal forces, due to drum rotations. Obviously the clothes final humidity level is higher than a dryer: typical water content percentage in garments after an high speed rinsing phase is not less than $45 \%$ (weight percentage), whilst in dryers load humidity is lowered down to room humidity values (for the most common fibers, a typical range is 3-8\%)

Same year later after the dryer market introduction, everywhere the need to reduce as much as possible the time between the washing phase and the ironing one has led to the introduction of wash-and-dryer machines, briefly indicated as WD. The possibility to combine both the washing action of a typical washing machine and the drying action performed in a dryer seemed to be consumerappealing mainly because of space availability in modern houses and ease of use. In these specific washing machines, water extraction operation has become a primary function as well as the washing one, as clearly defined by their name. The technical solution implemented in common WD is the same that we can find inside a dryer, the air circuit being optimized to the internal space availability of a washing machine. WD can be considered as a typical example of an already known solution optimizing based approach: the final result is obtained adapting a previously experienced one. This is a really common strategy in industry and even if it could seem to be effective, it does not help researchers and engineers to burst their well assessed framework in order to find other successful solutions out if it.

In this context the use of TRIZ models has been seen as a way to ensure goaloriented result, to discipline action for solutions to problems and to help in developing the capability to quickly recognize real possibilities or limitations during generation phase. The company main purpose was to find other technical solutions to dry clothes, in order to realize a more energetically efficient cycle and to obtain the improvements listed in chapter 3 . To pursuit these objectives, we have followed two building boxes of TRIZ theory: contradictions and, the maximal use of resources [8]. We have started our tests from a general model of the problem, considering as a main purpose, the introduction of "dry module" inside a commercial washing machine. The final output should be an energy efficient product in which both drying and washing function are considered as primary ones. The analysis has been carried on discussing about clothes and washing machines, but always taking into account company targets: finding a universal technical solution applicable to all household appliances which implement a drying cycle. For this reason, to find the best technical solution, we have firstly evaluated any possible resources present in the system (the washing machine), in its surrounding, or in the environment: before introducing new components or complications into the system, it is better to optimize already 
available resources. For each resource founded during the analysis, characteristic parameters have been identified. The marketing analysis previously discussed has been useful to define the "initial situation" of the problem enabling the identification of some system disadvantages that should have to be eliminated or the requirements through which improve the technique. The "Contradiction Matrix" use has been as a really useful tool to overcome these drawbacks and find the most effective solution instead of making usual engineering trade-offs. Patent literature analysis has been helpful both in the first both in last phase of our test. Several interesting results have been obtained, involving not only the household appliances area but also other correlated industrial activities showing the potentiality of TRIZ theory and its methodologies. Different processes and appliances have been considered as a single concept, which is, as already mentioned, "restoring the initial conditions" of a clean and dry item: TRIZ evidently demonstrated that clothes washing phase and crockery drying phase can be efficiently analyzed as specific situations of a more general model.

\subsection{The Resources analysis}

Being a washing machine the starting point of our test, the first resource is represented by the water itself, which can be classified as a natural or environmental one in accord to $[7,8]$. In this case the most important identified variables are: quantity of water and temperature. Water quantity is now, in modern washing machine, the optimal value for the washing phase both from energetic and clothes cleaning performance point of view. Also temperature is a crucial parameter, being straightly correlated to several factors: detergent activation, bacteria elimination, garments care. Such as for water, another important resource is represented by clothes, or material as a general meaning. Also in this case we have found two characteristic parameters: the amount of material to be washed (kilograms) and the fiber typology. In this case we can stand that these two parameters are really random and unknown during design phase because they dramatically depends upon customer needs and specific habits.

An additional resource is represented by the detergent, which has to perform the complex chemiophysical actions of removing stains from clothes. Regarding this resource the most representative parameters are the physical state (powder or liquid) and the chemical composition; both of them can significantly affect washing efficiency. Analyzing the system from an energy point of view, we can identify the electrical field which is used to turn on all the electrical equipment. For the electrical field the representative parameter is the power consumption, in terms of $\mathrm{kW}$. Other incoming energies can be used, such as natural gas, but electric power is today the most adopted solution. All the parameters identified are listed in table 1. Obviously during this analysis we have considered only resources which are already present inside the system after the washing phase because our main objective is to introduce an additional primary function inside an already 
existing system. The fact of considering only these resources it is not reductive for the methodology, because one of the possible technical solution could consist in giving auxiliary function to existing resources: it is only when all resources have been exhausted or it is impractical to utilize, that the consideration for additional design elements comes to play a crucial role. Technical solutions that use empty space or voids, waste products, byproducts, or harmful substances and fields, as well as available and very cheap energies and substances, can be significantly efficient.

Table 1. The Resource Analysis: parameters identification.

\begin{tabular}{ll}
\hline Resource & Parameters \\
\hline Water & - quantity (liters) \\
& - temperature $\left({ }^{\circ} \mathrm{C}\right)$ \\
\hline Clothes (Material) & - quantity (kilograms) \\
& - fiber typology \\
\hline Soap & - chemical compositions \\
& - physical state \\
\hline Electrical Energy & - Power $(\mathrm{kW})$ \\
\hline
\end{tabular}

\subsection{Technical problem solving: the Contradiction Matrix}

Once having defined the main system parameters we have applied contradictions analysis using "The Contradictions Matrix" (CM): a simplified CM has been built, by considering the reduced range of engineering parameters previously identified, and assuming the most suitable inventing principles. The decision of using this tool has been taken according to TRIZ theory which stands that the most effective inventive solution of a problem is the one that overcomes contradictions. To apply this tool we have considered the drying phase as the TP (technical process), and then we have analyzed contradictions among these parameters and the ones, that we have considered the most interesting for the case, selected by Altshuller between the late 1940s and 1970s $[6,11]$. To select these ones we have made a point of the results obtained from marketing researches already discussed in chapter 3 from which it clearly stood out that the waste in term of time, energy and power, together with harmful effects in general were the most relevant for customers.

The parameter "quantity of water" can be easily identified with "Amount of substance", the $26^{\text {th }}$ EP. In order to facilitate the water extraction, the amount of this substance should be as less as possible. The problem is that reducing the water could cause harmful effects, (31 EP) because the amount of water is optimized in order to have the best washing conditions. Another important contradiction came to light considering full-load washing cycle which needs a bigger amount of 
water. In this case this amount has negative effects on the power (21 EP) consumption as well as the energy spent by the subsystem (19-20 EP) and the time (25 EP) to perform the drying action. As well mentioned above, water temperature value (17 EP) is strictly connected to clothes fiber type and to the detergent chemical formulation. The increasing of this value, while can give to water more energy to came out from clothes fiber, can cause harmful from the fiber care point of view and requires a huge amount of power (21 EP). Contradiction analysis results are summarized in table 2 .

Table 2. Water analysis: the inventive principles.

\begin{tabular}{lcccc}
\hline & $\begin{array}{c}\text { Power } \\
(21)\end{array}$ & $\begin{array}{c}\text { Energy } \\
(19-20)\end{array}$ & $\begin{array}{c}\text { Spent Waste of Time } \\
(25)\end{array}$ & $\begin{array}{c}\text { Harmful Effects } \\
(31)\end{array}$ \\
\hline $\begin{array}{l}\text { Amount of } \\
\text { substance (26) }\end{array}$ & 35 & $3,35,31$ & $35,38,18,16$ & $3,35,40,39$ \\
\hline Temperature (17) & $2,14,17,25$ & & & $22,35,2,24$ \\
\hline
\end{tabular}

Several interesting technical solutions have been identified by means of the inventive principles analysis. Once having analysed these ones we found out that the most interesting ones could be classified in three different approaches: a better use of resources during the drying phase, the application of different physical fields (mechanical vibration or thermal phenomena) and the waste resources reuse after the drying phase.

For the first group of solutions we have:

1. Change the degree of flexibility (35)

2. Use only the necessary part (or property) of an object (2)

3. Using a multy-story arrangements of objects (17)

4. Using "slightly less" or "slightly more" of the same method (16)

Principle 35 suggests modifying the amount of water in relation to the amount and fibers typology of clothes in order to reduce power consumption: accordingly to this approach, the water to be eliminated is the only one useful for washing. This solution is already applied in common washing machine using special load sensors. The multi-story arrangements (17) and the use of only necessary part (2) could mean the creation of special drying cycle in relation to special washing ones that the consumer can choose. This is due to the fact that the clothes fibers type heavily influences both cycles: natural fibers like wool or cotton absorb a bigger quantity of water than synthetic ones and need a different water temperature value. For example another possible solution is to separate during drying phase the clothes already dried from the ones that still need additional treatment. The last inventive solutions (16) suggest precisely identifying the target humidity level to pursuit.

For the second group (mechanical vibration or thermal phenomena) we have:

1. Change an object's physical aggregate state (35) 
2. Go from linear to rotary motion, use centrifugal forces (14)

3. Tilt or re-orient the object (17)

4. Used combined ultrasonic or electromagnetic field oscillations (18)

Also inside this group of solutions we can find new ones and others nowadays applied. The solution already present in the market can be associated to the $35^{\text {th }}$ principle, due to the water physical aggregate state change realized by the hot flow. About this thermal phenomenon, a possible solution to facilitate water extraction could be the realization of a vacuum atmosphere inside the tank in order to reduce the energy spent by the system. Also the $14^{\text {th }}$ principle is already applied in washing machine as the classical mechanical solution, and it could be only optimized by developing specific rotating cycles. The last technical solution (18) has been considered as the most interesting and really innovative one. This solution principle is to transmit vibration energy to water molecules in a more efficient way than today, in order to facilitate the water extraction from clothes. Moreover, the $17^{\text {th }}$ principle could be read as a suggestion to optimize garments position at the beginning of the drying phase: being able to "unwrap" washed clothes and increase garments external surface would significantly improve drying process efficiency.

Finally in the third group has been classified these principles.

1. Use harmful factors to achieve a positive effect (22)

2. Make an object serve itself by performing auxiliary function (25)

3. Use waste resources, energy, or substances (25)

In this case technical solutions point an accent into the reuse of waste resources, e.g. extracting hot water by condensing it from the hot air flow, whose humidity value is usually extremely high, and reusing it to warm up cold one.

For clothes, detergent, and electrical energy parameters we do not have applied contradiction analysis. Clothes quantity value and fibers type can be considered as random variables because they depend on human needs. In reality there is a way through which enterprise can reduce this variability, to be more precise, identifying lots of specific washing and drying cycles as above mentioned in the first group of inventive solutions. Regarding to detergent, the possibility to facilitate the water physical aggregate state change could be realized with exothermic reactions. In this case the technical solution involves another market product which is not developed inside the company. Regarding to electrical field, this resource can be included into the technical solutions previously identified.

In conclusion, three possible problem approaches stand out from our analysis which can also be implemented concurrently. Identified solutions are based on a general approach so that they could be developed not only for a specific domestic appliance, like a washing machine, but also for all the ones which implement a drying cycle. Moreover the contraction analysis has seemed to be really effective in managing kick-off project meetings giving a well framed way to cope with technical problems. 


\subsection{The use of patent literature during concept design}

The strong advantage of TRIZ [12], in comparison with the other approaches, lies in Altshuller's choice of the right source for acquiring knowledge - the patent search and analysis. Patent information was the work basis, not only to create and introduce new procedures for the elimination of technical contradictions but also to examine general laws for the development of technical systems.

The Contradiction Matrix and the Principles do not work by themselves; they only suggest the most promising directions for engineers to search for a solution. The problem solver has to interpret these suggestions and determine how apply them to any particular situation. Nevertheless, TRIZ heuristics are very valuable because a problem solver does not have to study all patents from all disciplines, if he or she can reformulate the problem in the format of the Contradiction Matrix, thereby increasing the efficiency of solving. Regarding this, after having identified a sufficient number of possible contradictions solutions, we have started patent literature search. In particular we have focused our attention into microwave and ultrasound solutions.

Two different patent literature researches have been performed; the first at the beginning of the analysis considering a list of key-words like: to dry, water removing evaporation and humidity reduction. They have been generated only focusing on the product primary function. Obviously this research has lead to a big amount of patents because too generic but it has been useful to limit the research area. The second patent test has been conducted after the matrix contradictions analysis. Using words come out during technical contradictions solutions we have been able to limit progressively the analysis. A strong company commitment has given to pursuit a deeper involvement of patent literature analysis, also during the preliminary phases of innovation project: by systematically introducing specific TRIZ-based methodologies, significant and helpful outputs from patent analysis could be obtained in idea generation.

\section{Conclusions}

Problem solving lies at the heart of a new methodology for innovation. Innovators solve problems by focusing both upon the useful parameters of a system that, if increased, would enhance it substantially, and upon the harmful aspects that, if left unchecked, would lead to a contradiction. Contradictions are significant, because they contribute to the development of a breakthrough solution: avoiding compromise is central to innovation.

In this paper we present an industrial case study, whose beginning phase has been carried out to show TRIZ theory potentialities. By means of TRIZ Contradictions Matrix, interesting solution directions have been displaced. After evaluation, a set of innovative and feasible concepts have been obtained to solve 
the initial problem, the drying phase energy efficiency improvement of household appliances. In particular three different types of solution approaches have been identified which represent three different points of view to address the problem. The potentiality of identified solutions lies in the possibility to develop them for all the domestic appliances which have a drying cycle.

The suggested technical solutions have been deeply evaluated by company technical division, being two of them now under evaluation: specific internal projects are being developed to assess the technical feasibility of the proposed concepts. Furthermore, thanks to the interesting results obtained Indesit Company is now evaluating, on one side, the initiative of organizing idea generation meetings, in order to achieve a more effective involvement of all its innovation division engineers, not only middle and top management, and to maintain employee interest levels and participation rates, aligned to company innovation values. On the other side, further in-depth examinations will be carried out about patents search. In order to reach these purposes a deeper analysis of this theory and its methodology is fundamental because its potentialities in industry also depend on the personal properties of individuals and on their experience in applying it. For this reason several additional case studies will be implemented and analyzed.

\section{References}

1. Porter M. E. Stern S.: The New Challenge to America's Prosperity: Findings from the Innovation Index, Council on Competitiveness, Washington, DC (1999).

2. Kim W. C., Mauborgne R.: Strategy, value innovation, and the knowledge economy. Sloan Management Review, pp. 41-54, Springer (1999).

3. Harrington H. J., Continuous versus breakthrough improvement: finding the right answer. Business Process Reengineering \& Management Journal, vol. 1(3), pp. 3149 (1995).

4. Savransky S. D.: Introduction to TRIZ Methodology of Inventive Problem Solving, CRC Press, New York (2000).

5. Cascini G.: State of the art and trends of Computer Aided Innovation tools, towards the integration within the Product Development Cycle. In Building the information society, Kluwer Academy Publishers (ISBN 1-4020-8156-1) (2004).

6. Altshuller G. S.: Creativity as an Exact Science: The Theory of the Solution of Inventive Problems, Gordon and Breach Science Publishing, New York (1984).

7. Orloff M. A.: Inventive Thinking through TRIZ. A Practical Guide, pp 28-45, Springer (2006).

8. Mc Munigal J., Ungvari S., Slocum M., McMunigal R.: Mechanical Engineers Handbook: Materials and Mechanical Design, vol. 1, John Wiley \& Sons, Third Edition (2006).

9. Pevzner L. Kh.: ABC of Invention, Sredne-Ural'skoe Publishing House, Ekaterinburg (1992).

10. Sklobovsky K. A., Sharipov R. H.: Theory, Practice and Applications of the Inventive Problems Decision, Protva-Prin, Obninsk (1995).

11. Altshuller G. S.: 40 Principles: TRIZ Keys to Technical Innovation, TIC, Worchester (1998). 